\title{
A Review on Robust Watermarking with its Applications and Comparative Analysis
}

\author{
Gaurav Tiwari \\ Department of CSE/ IT \\ ITM, Gwalior, India \\ gaurav02tiwari@gmail.com
}

\begin{abstract}
Due to improvement in imaging skill and the ease with which digital content can be imitated and operated there is a strong requirement for a digital patent device to be put in place. It requires for authentication of the content as well as the owner. Digital Watermarking is present as a potential key to this problem. Till date several watermarking techniques have been proposed. This paper proposed a comprehensive survey of the current schemes that have been developed and their effectiveness.
\end{abstract}

Keywords: Applications, Transforms, Watermarking, Attacks, DCT, DFT, DWT

\section{Introduction}

In recent years, as digital media [2] are gaining wider popularity, their security related issues are becoming greater concern. Digital watermarking is a technique which allows an individual to add copyright notices or other verification messages to digital media. Image authentication is one of the applications of digital watermarking, which is used for authenticating the digital images. The objective is not to protect the contents from being copied or stolen, but is to provide a method to authenticate the image and assure the integrity of the image. The major drawback of digital signature is that it can detect if an image has been modified, but it cannot locate the regions where the image has been modified. To solve this problem, many researchers have proposed watermarking based schemes for image authentication.

\section{Watermarking Principle}

A watermarking mechanism is divided into three different steps-embedding, attack and detection. In embedding process, an algorithm takes the host and the data to be embedded and obtains secreted signals. The secreted signal is then communicated, usually communicated to another person. If this person creates a modification, this is called an attack. There are several possible attacks such as noise, blurring, etc. Detection is an algorithm which is applied to the attacked signal to try to extract the watermark from it. If the signal was not modified during transmission, then the watermark is still present and it can be extracted. If the signal is imitative, then the information is also carried in the copy. The embedding takes place by working the content of the digital data, which means the info is not embedded in the frame around the data, it is carried with the signal itself. The original image and the desired watermark are embedded using one of the various schemes that are currently available. The obtained watermarked image is passed through a decoder in which usually a reverse process to that employed during the embedding stage is applied to retrieve the watermark. The different techniques differ in the way in which it embeds the watermark on to the cover object. A secret key is used during the embedding and the extraction process in order to prevent illegal access to the watermark. 


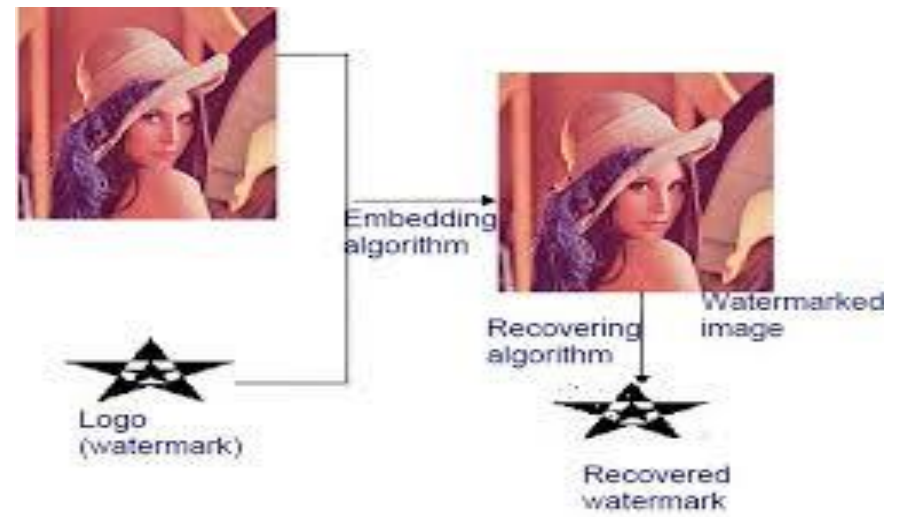

(General Overview of Watermarking Process)

\section{Ease of Use}

The template is used to format your paper and style the text. All margins, column widths, line spaces, and text fonts are prescribed; please do not alter them. You may note peculiarities. For example, the head margin in this template measures proportionately more than is customary. This measurement and others are deliberate, using specifications that anticipate your paper as one part of the entire proceedings, and not as an independent document. Please do not revise any of the current designations.

\section{Prepare Your Paper before Styling}

Before you begin to format your paper, first write and save the content as a separate text file. Keep your text and graphic files separate until after the text has been formatted and styled. Do not use hard tabs, and limit use of hard returns to only one return at the end of a paragraph. Do not add any kind of pagination anywhere in the paper. Do not number text heads - the template will do that for you.

Finally, complete content and organizational editing before formatting. Please take note of the following items when proofreading spelling and grammar.

\section{A. Abbreviations and Acronyms (Heading 2)}

Define abbreviations and acronyms the first time they are used in the text, even after they have been defined in the abstract. Abbreviations such as IEEE and SI do not have to be defined. Do not use abbreviations in the title or heads unless they are unavoidable.

\section{B. Units}

- Use either SI or CGS as primary units. (SI units are encouraged.) English units may be used as secondary units (in parentheses). An exception would be the use of English units as identifiers in trade, such as "3.5-inch disk drive".

- Avoid combining SI and CGS units, such as current in amperes and magnetic field in oersteds. This often leads to confusion because equations do not balance dimensionally. If you must use mixed units, clearly state the units for each quantity that you use in an equation.

- Do not mix complete spellings and abbreviations of units: " $\mathrm{Wb} / \mathrm{m}^{2}$ " or "webers per square meter", not "webers $/ \mathrm{m}^{2}$ ". Spell out units when they appear in text: ". . . a few henries", not ". . . a few H".

- Use a zero before decimal points: " 0.25 ", not “. 25 ". Use “cm", not "cc". (bullet list) 


\section{Equations}

The equations are an exception to the prescribed specifications of this template. You will need to determine whether or not your equation should be typed using either the Times New Roman or the Symbol font (please no other font). To create multileveled equations, it may be necessary to treat the equation as a graphic and insert it into the text after your paper is styled.

Number equations consecutively. Equation numbers, within parentheses, are to position flush right, as in Eq. 1, using a right tab stop. To make your equations more compact, you may use the solidus ( / ), the exp function, or appropriate exponents. Italicize Roman symbols for quantities and variables, but not Greek symbols. Use a long dash rather than a hyphen for a minus sign. Punctuate equations with commas or periods when they are part of a sentence, as in

$$
\alpha+\beta=\chi .
$$

Note that the equation is centered using a center tab stop. Be sure that the symbols in your equation have been defined before or immediately following the equation. Use "Eq. 1" or "Equation 1", not "(1)", especially at the beginning of a sentence: "Equation 1 is ..."

\section{Some Common Mistakes}

- The word "data" is plural, not singular.

- The subscript for the permeability of vacuum $\mu_{0}$, and other common scientific constants, is zero with subscript formatting, not a lowercase letter "o".

- In American English, commas, semi-/colons, periods, question and exclamation marks are located within quotation marks only when a complete thought or name is cited, such as a title or full quotation. When quotation marks are used, instead of a bold or italic typeface, to highlight a word or phrase, punctuation should appear outside of the quotation marks. A parenthetical phrase or statement at the end of a sentence is punctuated outside of the closing parenthesis (like this). (A parenthetical sentence is punctuated within the parentheses.)

- A graph within a graph is an "inset", not an "insert". The word alternatively is preferred to the word "alternately" (unless you really mean something that alternates).

- Do not use the word "essentially" to mean "approximately" or "effectively".

- In your paper title, if the words "that uses" can accurately replace the word "using", capitalize the " $u$ "; if not, keep using lower-cased.

- Be aware of the different meanings of the homophones "affect" and "effect", "complement" and "compliment", "discreet" and "discrete", "principal" and "principle".

- Do not confuse "imply" and "infer".

- The prefix "non" is not a word; it should be joined to the word it modifies, usually without a hyphen.

- There is no period after the "et" in the Latin abbreviation "et al.".

- The abbreviation "i.e." means "that is", and the abbreviation "e.g." means "for example".

An excellent style manual for science writers is given by Young [7].

\section{Using the Template}

After the text edit has been completed, the paper is ready for the template. Duplicate the template file by using the Save As command, and use the naming convention prescribed by your conference for the name of your paper. In this newly created file, highlight all of the contents and import your prepared text file. You are now ready to style your paper; use the scroll down window on the left of the MS Word Formatting toolbar. 


\section{E. Authors and Affiliations}

The template is designed so that author affiliations are not repeated each time for multiple authors of the same affiliation. Please keep your affiliations as succinct as possible (for example, do not differentiate among departments of the same organization). This template was designed for two affiliations.

1) For Author/s of Only One Affiliation (Heading 3): To change the default, adjust the template as follows.

a) Selection (Heading 4): Highlight all author and affiliation lines.

b) Change Number of Columns: Select Format > Columns >Presets > One Column.

c) Deletion: Delete the author and affiliation lines for the second affiliation.

2) For Authors of More than Two Affiliations: To change the default, adjust the template as follows.

a) Selection: Highlight all author and affiliation lines.

b) Change Number of Columns: Select Format > Columns > Presets > One Column.

c) Highlight Author and Affiliation Lines of Affiliation 1 and Copy this Selection.

d) Formatting: Insert one hard return immediately after the last character of the last affiliation line. Then paste down the copy of affiliation 1 . Repeat as necessary for each additional affiliation.

e) Reassign Number of Columns: Place your cursor to the right of the last character of the last affiliation line of an even numbered affiliation (e.g., if there are five affiliations, place your cursor at end of fourth affiliation). Drag the cursor up to highlight all of the above author and affiliation lines. Go to Format > Columns and select "2 Columns". If you have an odd number of affiliations, the final affiliation will be centered on the page; all previous will be in two columns.

\section{F. Identify the Headings}

Headings, or heads, are organizational devices that guide the reader through your paper. There are two types: component heads and text heads.

Component heads identify the different components of your paper and are not topically subordinate to each other. Examples include Acknowledgments and References and, for these, the correct style to use is "Heading 5". Use "figure caption" for your Figure captions, and "table head" for your table title. Run-in heads, such as "Abstract", will require you to apply a style (in this case, italic) in addition to the style provided by the drop down menu to differentiate the head from the text.

Text heads organize the topics on a relational, hierarchical basis. For example, the paper title is the primary text head because all subsequent material relates and elaborates on this one topic. If there are two or more sub-topics, the next level head (uppercase Roman numerals) should be used and, conversely, if there are not at least two sub-topics, then no subheads should be introduced. Styles named "Heading 1", "Heading 2", "Heading 3", and "Heading 4" are prescribed.

\section{G. Figures and Tables}

Place figures and tables at the top and bottom of columns. Avoid placing them in the middle of columns. Large figures and tables may span across both columns. Figure captions should be below the figures; table captions should appear above the tables. Insert figures and tables after they are cited in the text. Use the abbreviation "Figure 1" in the text, and "Figure 1" at the beginning of a sentence.

Use 8 point Times New Roman for figure labels. Use words rather than symbols or abbreviations when writing figure-axis labels to avoid confusing the reader. As an example, write the quantity "Magnetization", or "Magnetization, M", not just "M". 
If including units in the label, present them within parentheses. Do not label axes only with units. In the example, write "Magnetization $(\mathrm{A} / \mathrm{m})$ " or "Magnetization $\{\mathrm{A}[\mathrm{m}(1)]\}$ ", not just "A/m". Do not label axes with a ratio of quantities and units. For example, write "Temperature (K)", not "Temperature/K".

\section{H. Footnotes}

Use footnotes sparingly (or not at all) and place them at the bottom of the column on the page on which they are referenced. Use Times 8-point type, single-spaced.

To help your readers, avoid using footnotes altogether and include necessary peripheral observations in the text (within parentheses, if you prefer, as in this sentence).

Number footnotes separately from reference numbers, and in superscripts. Do not put footnotes in the reference list. Use letters for table footnotes.

Table 1. Table Type Styles

\begin{tabular}{|l|l|l|l|}
\hline $\begin{array}{c}\text { Table } \\
\text { Head }\end{array}$ & \multicolumn{3}{|c|}{ Table Column Head } \\
\hline & Table column subhead & Subhead & Subhead \\
\hline copy & More table copy $^{\mathrm{a}}$ & & \\
\hline
\end{tabular}

We suggest that you use a text box to insert a graphic (ideally $300 \mathrm{dpi}$, with all fonts embedded) because, in an MSW document, this method is somewhat more stable than directly inserting a picture.

To have non-visible rules on Example of a figure caption. (figure caption) your frame, use the MSWord pull-down menu, select Format > Borders and Shading > Select "None".

a. Sample of a table footnote. (table footnote)

Figure 1. Example of a Figure Caption; (figure caption)

\section{Conclusion}

In this paper, we provide a comprehensive survey on various digital watermarking techniques, their requirements, their properties, types and applications. Digital watermarking research has commonly concerned on two types of watermarks, fragile and robust. Robust watermarks are made to be detected even after attempts are made to remove them. Fragile watermarks are used for verification purposes and are capable of detecting even minute variations of the watermarked content. But neither type of watermark is ideal when considering "information preserving" transformations which reserve the sense or expression of the content and "information altering" transformations which change the look of the content. To solve this difficulty a semi fragile watermark for still images that can detect information altering transformations even after the watermarked content is subjected to information preserving alterations has to be used.

\section{References}

[1] D. Arya, "A Survey of Frequency and Wavelet Domain Digital Watermarking Techniques," International Journal of Scientific \& Engineering Research, November-2010 1 ISSN, vol. 1, no. 2, (2010) November, pp. 2229-5518

[2] S. Mishra, A. Mahapatra and P. Mishra, "A Survey on Digital Watermarking Techniques", vol. 4, no. 3, (2013), pp. 451-456. 
International Journal of Signal Processing, Image Processing and Pattern Recognition

Vol.8, No.6 (2015)

[3] I. S. Jacobs and C. P. Bean, "Fine particles, thin films and exchange anisotropy," in Magnetism, vol. III, G. T. Rado and H. Suhl, Eds. New York: Academic, (1963), pp. 271-350.

[4] K. Elissa, "Title of paper if known," unpublished.

[5] R. Nicole, "Title of paper with only first word capitalized," J. Name Stand. Abbrev., in press.

[6] Y. Yorozu, M. Hirano, K. Oka, and Y. Tagawa, "Electron spectroscopy studies on magneto-optical media and plastic substrate interface," IEEE Transl. J. Magn. Japan, vol. 2, pp. 740-741, August 1987 [Digests 9th Annual Conf. Magnetics Japan, p. 301, 1982].

[7] M. Young, The Technical Writer's Handbook. Mill Valley, CA: University Science, (1989). 